\title{
Immunohistochemical Differentiation between Urothelial Papillomas and Papillary Neoplasms of Low Malignant Potential of the Urinary Bladder
}

\author{
Mohammed Alrashidy ${ }^{1}$, Aliaa Atef ${ }^{1 *}$,Tarek Abdel Baky ${ }^{2}$
}

\begin{abstract}
Background: Urothelial papilloma and non-invasive papillary carcinoma are common neoplasms of the urinary bladder. Distinguishing papillomas and papillary carcinomas, especially the low grade type, is often debatable on the basis of histological features alone. Materials and Methods: We investigated immunohistochemical expression of cytokeratin $20(\mathrm{CK20})$, p53, and Ki-67 in a group of 20 urothelial papilloma cases and 30 noninvasive papillary neoplasms of low malignant potential (PNLMP) of the urinary bladder. Whole tissue sections were examined. Results: Among the 30 carcinoma cases, $12(40 \%)$ showed strong reactivity for the whole panel, $16(53 \%)$ reacted positively for two markers, and $2(7 \%)$ reacted just to one of them. Ki-67 was considered positive in 27 cases $(90 \%)$ and p53 in $24(80 \%)$, CK20 showed positive reactivity in 21 cases $(70 \%)$. Only small percentages of papillomas were positive, and then only weakly. Conclusions: We concluded that the intense positivity of suspicious cells for at least one of these markers would confirm the presence of malignant changes and favours the diagnosis of carcinoma.
\end{abstract}

Keywords: Papillary neoplasm of low malignant potential - papilloma - CK20 - P53 - Ki67 - immunohistochemistry

Asian Pac J Cancer Prev, 17 (4), 1769-1772

\section{Introduction}

The papillary lesions of the urinary bladder vary from benign lesions with recognizable morphologic features that merit the benign categorization "papilloma" to lesions showing dysplastic or malignant changes. The spectrum of primary malignancies of the urinary bladder can vary from superficial, well differentiated disease, which does not significantly impact patient survival, to highly malignant tumours for which long-term survival may be dismal. The vast majority of primary bladder tumours are of transitional cell variety (90-95\%) (Mehta et al., 2015). The World Health Organization (WHO) published a classification for urothelial papillary tumors. This classification system recognizes papilloma, papillary neoplasm of low malignant potential (PNLMP), and low-grade papillary transitional cell carcinoma at the low-grade end of the transitional cell neoplasia spectrum. Distinguishing urothelial papilloma from papillary carcinomas especially low grade non invasive ones is sometimes difficult on the basis of histological features alone (Eble et al., 2004).

Papillomas can be seen grossly as small excrescences or delicate frond like lesions but in the same time, papillary carcinomas especially those of low malignant potential can also be barely visible small growths. In non invasive tumors, a sharp interface with underlying normal bladder wall is often apparent on sectioning (Cheng and Bostwick, 2011). On microscopic basis, the papillae may branch but the particularly complex branching of many papillary carcinomas usually is not seen. Additionally, the papillae ,in papillomas, are usually covered by fewer than seven layers of urothelium, which is less than those of papillary carcinomas. In many cases of PNLMP, papillae are not stratified to more than seven layers, they don't usually have cells with appreciable cytologic atypia. Mitotic activity which favors papillary carcinoma is not always detected (Fletcher, 2013).

Immunohistochemical and molecular markers might be used as an adjunct to histology to reach an accurate diagnosis of bladder lesions. Several markers that characterize the histology and cellular appearance of tumors have been implicated in this differential diagnosis (Helpap et al., 2003). Cytokeratin 20 (CK20), p53, and $\mathrm{Ki}-67$ are related either to neoplastic change or prognosis in urothelial proliferations. Aberrant CK20 expression in urothelial cells plus overexpression of p53 and Ki-67 are indicators of dysplastic change in urothelial mucosa. Thus, immunohistochemistry is a useful tool to confirm the diagnosis of papillary carcinoma and could be helpful in detection of early dysplastic changes or early low grade non invasive carcinoma (Mallofre et al., 2003). 
Cytokeratins are a family of intermediate filament polypeptides present in epithelial cells. Cytokeratin 20 (CK20) is an isotype which shows a limited pattern of expression in normal tissues, being restricted to the gastric and intestinal epithelium, urothelium, and Merkel cells. It has been suggested that the pattern of CK20 staining is a useful adjunct to morphology in the diagnosis of urothelial dysplasia. It has also been suggested that CK20 expression can predict malignant potential in low-grade transitional cell tumors, and therefore CK20 can be useful in defining treatment strategies for patients with these tumors (Alsheik et al., 2001).

$\mathrm{Ki}-67$ is a nuclear protein that is present during the G1, $\mathrm{S}, \mathrm{G} 2$, and M phases of cycling cells. Previous studies have demonstrated a significant correlation between the Ki-67 index and tumor grade and stage of urinary bladder cancer (Cina et al., 2001). Mutations of the p53 gene have been found in a wide variety of malignant neoplasms, including urothelial carcinomas (Tut et al., 2001).

Thus, the purpose of the present study is to investigate if the immunohistochemical expression of the panel CK20, p53, and Ki-67 in urothelium can be a tool for distinguishing the cases of urothelial papilloma from those of low malignant potential.

\section{Materials and Methods}

We studied 20 cases considered as urothelial papilloma of urinary bladder and 30 cases of PNLMP by two uropathologists. Samples were collected as formalin-fixed, paraffin-embedded tissue blocks, with HandE stained slides from the archive of the pathology department of faculty of medicine, Tanta university (38) or received as fresh specimens taken by cystoscopic biopsy (12). Immunohistochemistry was performed using the antigen retrieval (PBS buffer; $\mathrm{pH} 7.2$ ) performed by immunoperxoidase method on $4 \mu$-thick sections from formalin-fixed, paraffin-embedded blocks. Monoclonal antibody to Ki-67 (MIB-1; 1:200, Labvision Biotechnology), p53 (1:200, Labvision Biotechnology), and CK20 (1:200, Labvision Biotechnology) were used. Samples with known positive reactivity for each marker were used as positive controls. As a negative control, a section was processed in which the primary antibody was changed by PBS. CK20 expression was considered aberrant when there was cytoplasmic expression on

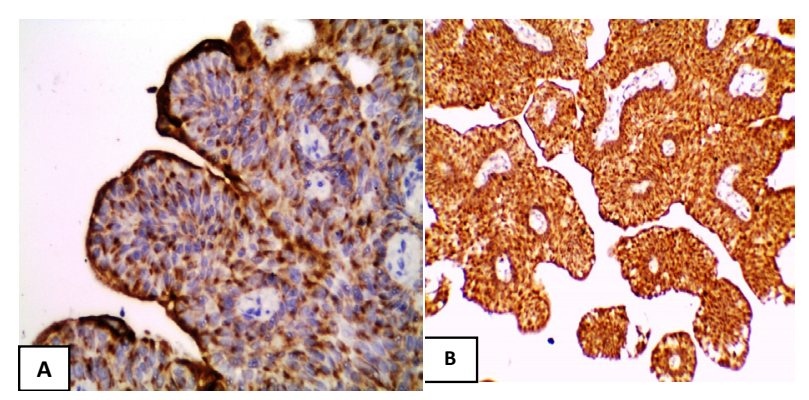

Figure 1. (A) Negative CK20 Expression in the Urothelial Cells of Urothelial Papilloma Except in the Umbrella Cells (X400). (B) Strong Positive Expression of CK20 in PNLMP (X100) urothelial cells other than superficial umbrella cells as nonneoplastic urothelium shows no reactivity to CK20 except for umbrella cells. Ki-67 was considered positive when $10 \%$ of cells showed nuclear positive expression and p53 was considered positive when $20 \%$ of cells showed nuclear positivity (Mallofre et al., 2003).

\section{Results}

In normal urothelial mucosa, nonneoplastic urothelium was negative for CK20 in the whole epithelium or expressed cytoplasmic positivity in a patchy way, just in umbrella cells. In the studied papilloma cases, all 20 cases (100\%) showed negative CK20 expression except for the umbrella cells (Figure 1(A)). P53 was expressed weakly in nuclei of some scattered cells, predominantly in a basal distribution, in 5 papilloma cases (25\%), in less than 5\% of cells (Figure 2(A)). Similarly, Ki-67 was negative in the whole urothelial cells of papilloma except for 6 cases

Table 1. Number of Markers Positive in PNLMPCases

\begin{tabular}{cc}
\hline Number of Positive Markers & PNLMP \\
\hline 1 & $2(7 \%)$ \\
2 & $16(53 \%)$ \\
3 & $12(40 \%)$ \\
\hline
\end{tabular}

Table 2. Number of Cases Positive for Each Marker in PNLMP Cases

\begin{tabular}{lcc}
\hline & \multicolumn{2}{c}{ Number of positive cases } \\
\hline & No. & $\%$ \\
CK20 & 20 & 67 \\
P53 & 24 & 80 \\
Ki67 & 26 & 87 \\
\hline
\end{tabular}
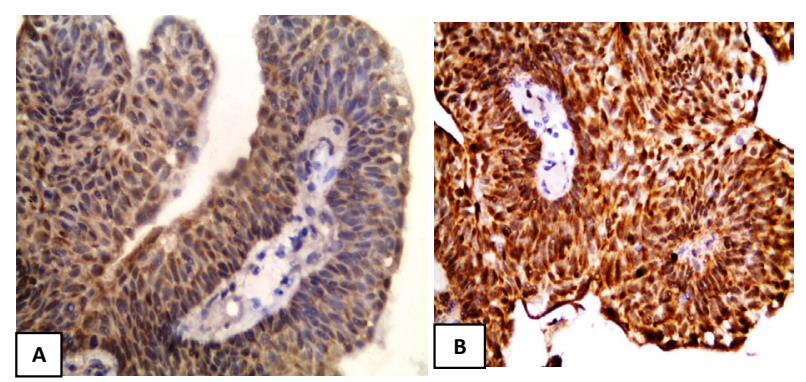

Figure 2. Weak Expression of P53 in Nuclei of Urothelial Papilloma (X400). (B) Strong Positive Expression of P53 in Nuclei of PNLMP (X400)'

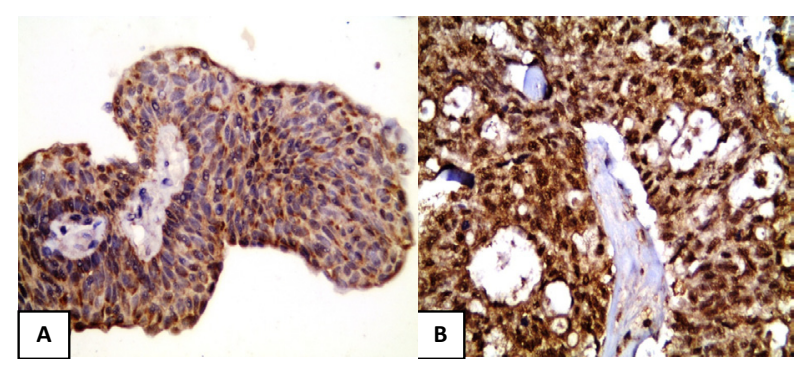

Figure 3. (A) Weak Nuclear Expression of Ki67 in Urothelial Papilloma (X200). (B) Strong Positive Ki67 Expression in Nuclei of PNLMP (X400). 
Immunohistochemical Differentiation between Urothelial Papilloma and Papillary Neoplasms of Low Malignant Potential

$(30 \%)$ expressed weak positivity in less than $5 \%$ of cells (Figure 3(A)).

On the other hand, The studied PNLMP cases showed a variable reactivity to the three markers. Among the 30 carcinoma cases, $12(40 \%)$ showed reactivity for the whole panel, $16(53 \%)$ reacted to two markers, and 2 (7\%) reacted just to one of them (Table 1). CK20 showed positive reactivity in 14 cases $(47 \%$ ) (Figure 1(B)), p53 was considered positive in 28 cases (93\%), (Figure 2(B)) and Ki-67 reacted in 28 cases (93\%) (Figure 3(B)) (Table $2)$.

\section{Discussion}

Neoplasms of the bladder pose biologic and clinical challenges. Despite significant inroads into their origins and improved methods of diagnosis and treatment, they continue to exact a high toll in morbidity and mortality. Papillary urothelial carcinoma is a common recurrent neoplasm with a significant number of cases in which neoplasm even the low grade ones progress to an infiltrating, very aggressive disease. There are cases in which cell pleomorphism is evident that it is easy to make a diagnosis. Nevertheless, there are still many cases in which pathologists do not feel absolutely confident in the diagnosis (Kumar et al., 2015).

Finding objective markers to aid in the distinction of true dysplastic changes from reactive cellular alterations is one of the challenges for uropathologists. Proliferation markers, oncogene products, adhesion molecules, and many other types of biological markers have been studied (Zaher and Sheridan, 2001). In this way, CK20 has been proposed as a marker of neoplastic change as well as a predictor for progression of urothelial carcinoma (Alsheik et al., 2001). Ki-67 is a proliferation marker, with demonstrated usefulness in many neoplasms in determining their progression and p 53 uniformly has been reported as a marker of progression in transitional cell carcinoma (Cina et al., 2001).

Our results of CK20 immunohistochemical expression were similar to those found by Mckenney et al, 2001. They found that, in the normal urothelium, CK20 showed patchy cytoplasmic immunoreactivity in only the superficial umbrella cell layer and nuclear immunoreactivity to p53 varied from negative to weak and patchy. In contrast, carcinoma in situ cases showed intense CK20 and p53 positivity ( $81 \%$ and $57 \%$, respectively) in the majority $(>50 \%)$ of malignant cells. They confirmed that these markers allow more confident distinction of urothelial CIS from non-neoplastic urothelium.

Mumtaz et al, 2014 also noticed diffuse positive expression of CK20 in carcinoma cases with $68.8 \%$ positivity in high grade cases and $40.4 \%$ positivity in low grade ones. Also, strong positive nuclear expression of p53 was found in $72.9 \%$ of high grade and in $36.2 \%$ of low grade cases. They stated that there was not only strong expression of both markers but also a significant difference in expression between low and high grade urothelial carcinoma.

In approval of our results, Mallofre et al. (2003), reached the same results as they stated that immunohistochemical panel composed of p53, Ki-67, and CK20 is useful for confirming the presence of dysplastic changes in the urothelium. The positivity was always strong and, in many cases, in a high percentage of cells (ranging from $50-90 \%)$. This immunohistochemical profile is clearly different from the one observed in normal urothelium, in which the whole panel is always negative or just weakly positive in few cells.

Pich et al, 2001, studied the expression of ki67 and p53 in urothelial carcinomas. They noticed increased expression of these two markers with increase of grade. Similarly, Bertz et al, 2014, confirmed that CK20, P53 and ki67 are reliable indicators of biologic aggressiveness in urothelial carcinomas. Besides, Krause et al, 2000, showed that benign tissues were negative for Ki67 and p53 while the superficial bladder cancer (Ta, T1) showed, in comparison to the invasive tumours, a higher rate of p53 mutations.

In contrast to the previous results, Van Oers et al, 2007, found that abnormal CK20 expression was strongly associated only with higher tumour grades and stages while low-grade noninvasive papillary tumours is defined by the presence of a normal CK20 expression pattern. Cina et al, 2001, examined p53 and Ki-67 staining patterns of the diagnostic entities included within the new World Health Organization/International Society of Urological Pathology (WHO/ISUP) classification of urothelial neoplasms. They found an increased proliferative index as demonstrated by immunohistochemical staining for Ki67 of papillary carcinoma and high grade intraepithelial neoplasia. Interestingly, marked p53 positivity was also characteristic of carcinoma but may be seen in benignappearing urothelium, suggesting a "field effect" with occult molecular aberration.

According to Yin and Leong, 2004, CK20, Ki-67, and p53 were applied to 84 noninvasive papillary urothelial tumors. In the WHO/ISUP classification, all benign lesions showed normal CK20 staining and all carcinomas showed abnormal staining. The Ki-67 index was significantly different between benign and malignant lesions $(\mathrm{P}<0.05)$ and between low- and high grade carcinomas $(\mathrm{P}<0.001)$. p53 was negative in all benign lesions, with a significant difference between low- and high-grade carcinomas (P < 0.001). By the 1973 WHO classification, normal CK20 staining was present both in benign lesions and in carcinomas. Ki-67 staining did not distinguish between grade 2 and grade 3 carcinomas $(\mathrm{P}>0.05)$, and there was no difference in $\mathrm{p} 53$ staining in grades 1 and 2 carcinomas ( $\mathrm{P}>0.05)$.

In conclusion, according to our results, The positivity of one or more of the three markers can be used as indicative of malignant change of urothelial cells and development of carcinoma even the PNLMP. From a differential diagnosis perspective, use of at least one of the three antibodies with morphologic correlation would be essential for accurate diagnosis of papillary urothelial neoplasms.

\section{Acknowledgements}

We wish to thank the Department of Pathology of 


\section{Mohammed Alrashidy et al}

Faculty of Medicine, Tanta University, for providing us with a large part of the cases from the archive of the department and making our work much easier.

\section{References}

Alsheik A, Mohamedali Z, Jones E, et al (2001). Comparison of the WHO/ISUP classification and cytokeratin expression in predicting the behaviour of low-grade papillary urothelial tumors. Mod Pathol, 14, 267-72.

Bertz S, Otto W, Denzinger S, et al (2014). Combination of CK20 and $\mathrm{Ki}-67$ immunostaining analysis predicts recurrence, progression, and cancer-specific survival in pT1 urothelial bladder cancer. European Urol, 65, 218-26.

Cheng L and Bostwick D (2011). Essentials of Anatomic Pathology. Pub. Springer Science and Business Media, $3^{\text {rd }}$ ed., Ch. 36.

Cina SJ, Lancaster-Weiss KJ, Lecksell K, et al (2001). Correlation of Ki67 and p53 with the new world health organization/international society of urological pathology classification system for urothelial neoplasia. Arch Pathol Lab Med, 125, 646-51.

Eble J, Sauter G, Epstein J and Sesterhenn I (2004). World health organization classification of tumours. pathology and genetics of tumours of the urinary system and male genital organs. Pub. IARC Press Lyon, France, 90-110.

Fletcher C (2013). Diagnostic histopathology of tumors. 4th ed., Pub. Elsevier Saunders, vol. 1, ch. 12, 610-11.

Helpap B, Schmitz-drager B, Hamilton P, et al (2003): Molecular pathology of non-invasive urothelial carcinomas (part 1). Virchows Arch, 442, 309-16.

Krause FS, Feil G, Bichler KH (2000): Immunohistochemical examinations (Ki67, p53, nm23) and DNA cytophotometry in bladder cancer. Anticancer Res, 20, 5023-8.

Kumar V, Abbas A, Aster J (2015): Robbins and Cotran Pathologic Basis of Disease, $9^{\text {th }}$ ed., Pub. Elseveir Saunders, ch. 21, 1028.

Mallofre C, Castillo M, Morente V, Sole M (2003). Immunohistochemical expression of CK20, p53, and Ki67 as objective markers of urothelial dysplasia. Mod Pathol, 16, 187-91.

McKenney JK, Desai S, Cohen C, Amin MB (2001). Discriminatory immunohistochemical staining of urothelial carcinoma in situ and non-neoplastic urothelium: an analysis of cytokeratin 20, p53, and CD44 Antigens. Am J Surgical Pathol, 25, 1074-8.

Mehta N, Rathore RS, Pillai BS, et al (2015). Intrinsic tumour factors affecting recurrence in non muscle invasive bladder cancer: a hospital based study from india. Asian Pac J Cancer Prev, 16, 2675-7.

Mumtaz S, Hashmi AA, Hasan SH, et al (2014). Diagnostic utility of p53 and CK20 immunohistochemical expression grading urothelial malignancies. Int Arch Med, 7, 36.

Pich A, Chiusa L, Formiconi A, et al (2001). Biological differences between noninvasive papillary urothelial neoplasms of low malignant potential and low-grade (grade 1) papillary carcinomas of the bladder. Am J Surg Pathol, 25, 1528- 33 .

Tut VM, Braithwaite KL, Angus B, et al (2001). Cyclin D1 expression in transitional cell carcinoma of the bladder: correlation with p53, waf1, pRb and Ki67. Br J Cancer, 84, 270-5.

Van Oers JM, Wild PJ, Burger M, et al (2007). FGFR3 Mutations and a Normal CK20 Staining Pattern Define Low-Grade Noninvasive Urothelial Bladder Tumours. European Urol, 52, 760-8.

Yin H, Leong A (2004). Histologic grading of noninvasive papillary urothelial tumors. validation of the $1998 \mathrm{WHO} /$ ISUP system by immunophenotyping and Follow-up. Am J Clin Pathol, 121, 679-87.

Zaher A, Sheridan T (2001). Tumor markers in the detection of recurrent transitional cell carcinoma of the bladder. Acta Cytol, 45, 575-81. 